\title{
Development of Race-Specific SCAR Markers for Detection of Chinese Races CYR32 and CYR33 of Puccinia striiformis f. sp. tritici
}

\author{
Baotong Wang, Xiaoping Hu, Qiang Li, Baojun Hao, Bo Zhang, Gaobao Li, and Zhensheng Kang, College of \\ Plant Protection and Shaanxi Key Laboratory of Molecular Biology for Agriculture, Northwest A\&F University, \\ Yangling, 712100, China
}

\begin{abstract}
Wang, B., Hu, X., Li, Q., Hao, B., Zhang, B., Li, G., and Kang, Z. 2010. Development of racespecific SCAR markers for detection of Chinese races CYR32 and CYR33 of Puccinia striiformis f. sp. tritici. Plant Dis. 94:221-228.

Wheat stripe rust, caused by Puccinia striiformis f. sp. tritici, is a devastating disease in China. Races CYR32 and CYR33 have been predominant in the recent $P$. striiformis $\mathrm{f}$. sp. tritici population. To develop molecular markers for these races, initially 86 isolates, most of which were collected in 2007 throughout China, were tested on the set of wheat genotypes for differentiating Chinese $P$. striiformis f. sp. tritici races, and their genomic DNA were amplified with 94 random amplified polymorphic DNA (RAPD) primers. Twelve isolates were identified as CYR33, 14 as CYR32, and 60 as 13 other races. A 320-bp band was identified to be associated with CYR32 with primer S1271 (5'-CTTCTCGGTC-3'), and a 550-bp band was identified to be specific to CYR33 with primer S1304 (5'-AGGAGCGACA-3'). The two bands were cloned and sequenced. Based on the sequences, sequence characterized amplified region (SCAR) markers CYR32sp1/sp2 and CYR33sp1/sp2 were developed to differentiate CYR32 and CYR33, respectively, from other races. The SCAR markers were validated with DNA samples from wheat leaves inoculated with selected isolates from the 86 isolates and urediniospore DNA samples from an additional 63 isolates collected from 2006 to 2009. The detection of CYR32 and CYR33 with the SCAR markers was completely consistent with the results of the race identification with the set of differential wheat genotypes. Thus, the markers are highly reliable for identification of the two races.
\end{abstract}

Stripe rust, caused by Puccinia striiformis Westend. f. sp. tritici Eriks., can result in severe economic losses for wheat growers in many countries $(5,15,29)$. In China, stripe rust is one of the most destructive diseases of wheat (21). The pathogen population is highly variable, and new virulent races can quickly become predominant and overcome race-specific resistance of wheat cultivars. So far, a total of $71 P$. striiformis $\mathrm{f}$. sp. tritici races have been identified, of which 33 were designated as CYR (for Chinese yellow rust) races and 38 as "pathotypes" with particular virulences on wheat differential genotypes Lovrin 10 (Lov10), Lovrin 13 (Lov13), Hybrid 46 (Hy46), and Suwon 11 (Su11) $(2,21)$. The frequencies of CYR32 and CYR33 were the highest among all detected races from 1997 to 2009 (21-24). With the major virulences in the current $P$. striiformis f. sp. tritici population, these two races have become the major targeting races for breeding wheat cultivars with effective resistance.

Corresponding and co-first authors: Baotong Wang, E-mail: wangbt@nwsuaf.edu.cn Xiaoping Hu, E-mail: xphu@nwsuaf.edu.cn

Accepted for publication 26 October 2009.

doi:10.1094/PDIS-94-2-0221

(C) 2010 The American Phytopathological Society
Because urediniospores of $P$. striiformis f. sp. tritici can be disseminated by wind for hundreds of kilometers in a few hours, stripe rust can develop quickly on a large scale. Thus, early detection of races is important for disease management. The current $P$. striiformis f. sp. tritici race identification mainly depends on the virulence phenotype of the pathogen on a set of wheat differential genotypes. This approach takes a couple of months because urediniospores from an original sample need to be increased to enough for testing on the set of differential genotypes in a conditioned greenhouse. Such testing can be limited by space and temperature conditions, and is also time-consuming and labor-intensive. Therefore, it is necessary to develop a simple, rapid, and effective technique for detection of $P$. striiformis $\mathrm{f}$. sp. tritici races.

Several molecular techniques have been used to differentiate species of Puccinia and formae speciales of $P$. striiformis. Newton et al. (17) used double-stranded RNA to separate formae speciales of $P$. striiformis. Shan et al. (19) used a moderately repetitive sequence from $P$. striiformis f. sp. tritici to identify 96 genotypes in the Chinese population. Polymerase chain reaction (PCR)-based approaches have the advantages of speed, less limitation by time and space, and sufficient sensitivity and specificity. Chen et al. $(6,7)$ used the random amplified polymorphic DNA (RAPD) technique to differentiate Puccinia species, formae speciales of $P$. striiformis, and races of $P$. striiformis f. sp. tritici in the United States. They separated race PST-21 from other races with several RAPD markers. More recently, amplified fragment length polymorphism (AFLP) and simple sequence repeat (SSR) markers have been used to study $P$. striiformis f. sp. tritici population structures and the spread of particular virulent strains $(3,8,11,12,16,20)$. The objective of this study was to develop specific markers for detecting the predominant $P$. striiformis f. sp. tritici races in China.

\section{MATERIALS AND METHODS}

Stripe rust sampling. Initially, 86 isolates of $P$. striiformis $\mathrm{f}$. sp. tritici were used in screening for RAPD markers associated with races CYR32 and CYR33. To validate the SCAR markers specific for CYR32 or CYR33, an additional 63 isolates were used. The 86 isolates in the first group were selected to represent 15 races (CYR22, CYR24, CYR29, CYR31, CYR32, CYR33, Hy46-8, Z4, Su11-1, Su11-3, Su11-4, Su11-5, Su11-6, Su11-7, and Su11-10) detected in 2006 and 2007 (4). They were mostly collected in 2007 from five provinces (Shaanxi, Gansu, Yunnan, Sichuan, and Henan) (Table 1). The 63 isolates in the second group were collected from 2006 to 2009 and identified as 10 races (CYR22, CYR24, CYR29, CYR30, CYR31, CYR32, CYR33, Su11-4, Su11-7, and Su11-11). All races of the Su11 series are virulent on wheat differential Suwon 11 and virulent or avirulent on other differential genotypes. Each isolate was increased on susceptible wheat genotype Mingxian 169 and tested on the set of 17 wheat genotypes used to differentiate Chinese $P$. striiformis f. sp. tritici races $(4,21,23)$. Ten single-spore isolates of CYR32 and five single-spore isolates of CYR33 were further obtained by reisolation after testing on the differentials. Urediniospores were vacuum-dried and kept at $-80^{\circ} \mathrm{C}$ until use.

Inoculation of wheat seedlings and race identification. Race identification was conducted using the standard procedures as previously described $(13,23)$. For confirming SCAR markers, seedlings of susceptible wheat genotype Mingxian 169 grown under controlled greenhouse 
Table 1. Isolates of Puccinia striiformis f. sp. tritici used in study and presence or absence of markers for races CYR32 and CYR33

\begin{tabular}{|c|c|c|c|c|c|c|}
\hline Isolate & Origin & Year & Race/pathotype $^{a}$ & Virulence formula $^{b}$ & CYR32sp1/sp2 ${ }^{\mathrm{c}}$ & CYR33sp1/sp2 \\
\hline \multicolumn{7}{|c|}{ Isolates used in random amplified polymorphic DNA marker identification } \\
\hline $07-5-12-4$ & Shaanxi & 2007 & CYR33 & $1,2,3,4,5,6,7,8,9,10,11,12,13,14,16$ & - & + \\
\hline $07-5-12-1$ & Shaanxi & 2007 & CYR33 & $1,2,3,4,5,6,7,8,9,10,11,12,13,14,16$ & - & + \\
\hline $07-5-4-2$ & Shaanxi & 2007 & CYR33 & $1,2,3,4,5,6,7,8,9,10,11,12,13,14,16$ & - & + \\
\hline $07-5-1-7$ & Shaanxi & 2007 & CYR33 & $1,2,3,4,5,6,7,8,9,10,11,12,13,14,16$ & - & + \\
\hline $07-5-3-6$ & Shaanxi & 2007 & CYR33 & $1,2,3,4,5,6,7,8,9,10,11,12,13,14,16$ & - & + \\
\hline $07-7-6-5$ & Gansu & 2007 & CYR33 & $1,2,3,4,5,6,7,8,9,10,11,12,13,14,16$ & - & + \\
\hline $07-7-2-13$ & Gansu & 2007 & CYR33 & $1,2,3,4,5,6,7,8,9,10,11,12,13,14,16$ & - & + \\
\hline $07-7-2-12$ & Gansu & 2007 & CYR33 & $1,2,3,4,5,6,7,8,9,10,11,12,13,14,16$ & - & + \\
\hline $07-7-4-3$ & Gansu & 2007 & CYR33 & $1,2,3,4,5,6,7,8,9,10,11,12,13,14,16$ & - & + \\
\hline $07-15-1-2$ & Yunnan & 2007 & CYR33 & $1,2,3,4,5,6,7,8,9,10,11,12,13,14,16$ & - & + \\
\hline $07-15-2-11$ & Yunnan & 2007 & CYR33 & $1,2,3,4,5,6,7,8,9,10,11,12,13,14,16$ & - & + \\
\hline $07-15-3-2$ & Yunnan & 2007 & CYR33 & $1,2,3,4,5,6,7,8,9,10,11,12,13,14,16$ & - & + \\
\hline $07-5-1-1$ & Shaanxi & 2007 & CYR32 & $1,2,3,4,5,6,7,8,9,10,11,12,13,14,16,17$ & + & - \\
\hline $07-5-2-3$ & Shaanxi & 2007 & CYR32 & $1,2,3,4,5,6,7,8,9,10,11,12,13,14,16,17$ & + & - \\
\hline $07-5-3-6$ & Shaanxi & 2007 & CYR32 & $1,2,3,4,5,6,7,8,9,10,11,12,13,14,16,17$ & + & - \\
\hline $06-5-6-15$ & Shaanxi & 2006 & CYR32 & $1,2,3,4,5,6,7,8,9,10,11,12,13,14,16,17$ & + & _- \\
\hline $06-5-8-3$ & Shaanxi & 2006 & CYR32 & $1,2,3,4,5,6,7,8,9,10,11,12,13,14,16,17$ & + & - \\
\hline $07-7-8-6$ & Gansu & 2007 & CYR32 & $1,2,3,4,5,6,7,8,9,10,11,12,13,14,16,17$ & + & - \\
\hline $07-7-6-4$ & Gansu & 2007 & CYR32 & $1,2,3,4,5,6,7,8,9,10,11,12,13,14,16,17$ & + & - \\
\hline $07-7-3-12$ & Gansu & 2007 & CYR32 & $1,2,3,4,5,6,7,8,9,10,11,12,13,14,16,17$ & + & - \\
\hline $07-7-6-8$ & Gansu & 2007 & CYR32 & $1,2,3,4,5,6,7,8,9,10,11,12,13,14,16,17$ & + & - \\
\hline $07-6-2-3$ & Sichuan & 2007 & CYR32 & $1,2,3,4,5,6,7,8,9,10,11,12,13,14,16,17$ & + & - \\
\hline $07-6-3-11$ & Sichuan & 2007 & CYR32 & $1,2,3,4,5,6,7,8,9,10,11,12,13,14,16,17$ & + & _- \\
\hline $07-2-4-2$ & Henan & 2007 & CYR32 & $1,2,3,4,5,6,7,8,9,10,11,12,13,14,16,17$ & + & _- \\
\hline $07-15-3-5$ & Yunnan & 2007 & CYR32 & $1,2,3,4,5,6,7,8,9,10,11,12,13,14,16,17$ & + & - \\
\hline $07-15-3-4$ & Yunnan & 2007 & CYR32 & $1,2,3,4,5,6,7,8,9,10,11,12,13,14,16,17$ & + & - \\
\hline $07-5-9-3$ & Shaanxi & 2007 & CYR31 & $1,2,3,4,5,6,7,8,9,11,12,14,16,17$ & - & _- \\
\hline $07-5-5-14$ & Shaanxi & 2007 & CYR31 & $1,2,3,4,5,6,7,8,9,11,12,14,16,17$ & - & - \\
\hline $07-5-3-16$ & Shaanxi & 2007 & CYR31 & $1,2,3,4,5,6,7,8,9,11,12,14,16,17$ & - & - \\
\hline $07-7-6-1$ & Gansu & 2007 & CYR31 & $1,2,3,4,5,6,7,8,9,11,12,14,16,17$ & - & - \\
\hline $07-7-2-4$ & Gansu & 2007 & CYR31 & $1,2,3,4,5,6,7,8,9,11,12,14,16,17$ & - & _- \\
\hline $07-5-10-2$ & Shaanxi & 2007 & CYR31 & $1,2,3,4,5,6,7,8,9,11,12,14,16,17$ & - & - \\
\hline $07-5-10-5$ & Shaanxi & 2007 & CYR31 & $1,2,3,4,5,6,7,8,9,11,12,14,16,17$ & - & - \\
\hline $07-5-8-3$ & Shaanxi & 2007 & CYR31 & $1,2,3,4,5,6,7,8,9,11,12,14,16,17$ & - & - \\
\hline $07-7-2-6$ & Gansu & 2007 & CYR31 & $1,2,3,4,5,6,7,8,9,11,12,14,16,17$ & - & _- \\
\hline $07-7-2-7$ & Gansu & 2007 & CYR31 & $1,2,3,4,5,6,7,8,9,11,12,14,16,17$ & - & - \\
\hline $07-5-1-12$ & Shaanxi & 2007 & CYR29 & $1,2,3,4,5,6,7,8,9,11,12,16$ & - & - \\
\hline $07-5-2-4$ & Shaanxi & 2007 & CYR29 & $1,2,3,4,5,6,7,8,9,11,12,16$ & - & - \\
\hline $07-5-6-3$ & Shaanxi & 2007 & CYR29 & $1,2,3,4,5,6,7,8,9,11,12,16$ & - & - \\
\hline $07-7-1-14$ & Gansu & 2007 & CYR29 & $1,2,3,4,5,6,7,8,9,11,12,16$ & - & - \\
\hline $07-7-2-5$ & Gansu & 2007 & CYR29 & $1,2,3,4,5,6,7,8,9,11,12,16$ & - & - \\
\hline $07-5-1-8$ & Shaanxi & 2007 & CYR22 & $1,2,3,4,5,6,7,8,9,10,11,13$ & - & _- \\
\hline $07-5-1-4$ & Shaanxi & 2007 & CYR22 & $1,2,3,4,5,6,7,8,9,10,11,13$ & - & - \\
\hline $07-5-1-11$ & Shaanxi & 2007 & CYR22 & $1,2,3,4,5,6,7,8,9,10,11,13$ & - & - \\
\hline $07-5-2-5$ & Shaanxi & 2007 & CYR24 & $1,2,3,4,6,7,8,9,11$ & - & - \\
\hline $07-5-3-8$ & Shaanxi & 2007 & CYR24 & $1,2,3,4,6,7,8,9,11$ & - & _- \\
\hline $07-5-3-10$ & Shaanxi & 2007 & CYR24 & $1,2,3,4,6,7,8,9,11$ & - & - \\
\hline $07-5-1-13$ & Shaanxi & 2007 & Нy $46-8$ & $1,2,3,4,5,6,7,8,9,10,11,12,14,16,17$ & - & - \\
\hline $07-5-3-1$ & Shaanxi & 2007 & Hy $46-8$ & $1,2,3,4,5,6,7,8,9,10,11,12,14,16,17$ & - & - \\
\hline $07-5-3-4$ & Shaanxi & 2007 & Нy $46-8$ & $1,2,3,4,5,6,7,8,9,10,11,12,14,16,17$ & - & - \\
\hline $07-5-5-4$ & Shaanxi & 2007 & $\mathrm{Z4}$ & $1,2,3,4,6,7,8,9,10,11,13,14,15$ & - & - \\
\hline $07-5-35-2$ & Shaanxi & 2007 & Su11-1 & $1,2,7,14$ & - & - \\
\hline $07-5-37-8$ & Shaanxi & 2007 & Su11-1 & $1,2,7,14$ & - & - \\
\hline $07-5-37-2$ & Shaanxi & 2007 & Su11-1 & $1,2,7,14$ & - & - \\
\hline $07-7-12-3$ & Gansu & 2007 & Su11-1 & $1,2,7,14$ & - & - \\
\hline $07-7-11-4$ & Gansu & 2007 & Su11-1 & $1,2,7,14$ & - & - \\
\hline $07-5-34-5$ & Shaanxi & 2007 & Su11-3 & $1,2,3,4,5,6,7,8,9,10,11,13,14$ & - & - \\
\hline $07-5-33-1$ & Shaanxi & 2007 & Su11-3 & $1,2,3,4,5,6,7,8,9,10,11,13,14$ & - & - \\
\hline $07-5-38-5$ & Shaanxi & 2007 & Su11-3 & $1,2,3,4,5,6,7,8,9,10,11,13,14$ & - & - \\
\hline $07-7-6-13$ & Gansu & 2007 & Su11-3 & $1,2,3,4,5,6,7,8,9,10,11,13,14$ & - & - \\
\hline $07-7-4-10$ & Gansu & 2007 & Su11-3 & $1,2,3,4,5,6,7,8,9,10,11,13,14$ & - & - \\
\hline $07-5-7-4$ & Shaanxi & 2007 & Su11-4 & $2,3,4,6,7,8,9,10,11,13,14,16$ & _- & _- \\
\hline $07-5-6-11$ & Shaanxi & 2007 & Su11-4 & $2,3,4,6,7,8,9,10,11,13,14,16$ & - & - \\
\hline $07-5-33-4$ & Shaanxi & 2007 & Su11-4 & $2,3,4,6,7,8,9,10,11,13,14,16$ & - & - \\
\hline $07-7-12-5$ & Gansu & 2007 & Su11-4 & $2,3,4,6,7,8,9,10,11,13,14,16$ & - & - \\
\hline $07-7-6-2$ & Gansu & 2007 & Su11-4 & $2,3,4,6,7,8,9,10,11,13,14,16$ & - & - \\
\hline $07-5-38-6$ & Shaanxi & 2007 & Su11-5 & $1,2,3,4,5,6,7,8,9,11,14,16$ & - & - \\
\hline $07-5-33-6$ & Shaanxi & 2007 & Su11-5 & $1,2,3,4,5,6,7,8,9,11,14,16$ & - & - \\
\hline $07-5-31-1$ & Shaanxi & 2007 & Su11-5 & $1,2,3,4,5,6,7,8,9,11,14,16$ & - & - \\
\hline $07-7-8-12$ & Gansu & 2007 & Su11-5 & $1,2,3,4,5,6,7,8,9,11,14,16$ & - & - \\
\hline $07-7-1-13$ & Gansu & 2007 & Su11-5 & $1,2,3,4,5,6,7,8,9,11,14,16$ & - & - \\
\hline
\end{tabular}

\footnotetext{
${ }^{a}$ CYR indicates races of Chinese yellow rust; Su11-X, Hy46-X, and Z4 indicate different "pathotypes" within race groups virulent on differential Suwon 11, Hybrid 46, and Zhong 4, respectively.

${ }^{\mathrm{b}}$ Chinese differential genotypes: $1=$ Trigo Eureka, $2=$ Fulhard, $3=$ Lutescens 128, $4=$ Mentana, $5=$ Virgilio, $6=$ Abbondanza, $7=$ Early Premium, $8=$ Funo, 9 $=$ Danish $1,10=$ Jubilejina 2, $11=$ Fengchan 3,12 = Lovrin 13, $13=$ Kangyin 655, $14=$ Suwon $11,15=$ Zhong 4, $16=$ Lovrin 10 , and $17=$ Hybrid 46.

${ }^{\mathrm{c}}+$ indicates present and - indicates absent.
} 
Table 1. (continued from previous page)

\begin{tabular}{|c|c|c|c|c|c|c|}
\hline Isolate & Origin & Year & Race/pathotype ${ }^{a}$ & Virulence formula $^{b}$ & CYR32sp1/sp2c & CYR33sp1/sp2 ${ }^{\mathrm{c}}$ \\
\hline $07-5-14-2$ & Shaanxi & 2007 & Su11-6 & $1,2,3,6,7,8,9,11,14$ & - & - \\
\hline $07-5-4-6$ & Shaanxi & 2007 & Su11-6 & $1,2,3,6,7,8,9,11,14$ & - & - \\
\hline $07-5-12-5$ & Shaanxi & 2007 & Su11-6 & $1,2,3,6,7,8,9,11,14$ & - & - \\
\hline $07-7-3-15$ & Gansu & 2007 & Su11-6 & $1,2,3,6,7,8,9,11,14$ & - & - \\
\hline $07-5-2-1$ & Shaanxi & 2007 & Su11-7 & $1,2,3,4,5,6,7,8,9,11,12,14,16$ & - & - \\
\hline $07-5-3-15$ & Shaanxi & 2007 & Su11-7 & $1,2,3,4,5,6,7,8,9,11,12,14,16$ & - & - \\
\hline $07-5-3-13$ & Shaanxi & 2007 & Su11-7 & $1,2,3,4,5,6,7,8,9,11,12,14,16$ & - & - \\
\hline $07-7-2-11$ & Gansu & 2007 & Su11-7 & $1,2,3,4,5,6,7,8,9,11,12,14,16$ & - & - \\
\hline $07-5-3-11$ & Shaanxi & 2007 & Su11-10 & $2,3,4,6,7,8,9,11,14$ & - & - \\
\hline $07-5-3-7$ & Shaanxi & 2007 & Su11-10 & $2,3,4,6,7,8,9,11,14$ & - & - \\
\hline $07-5-3-3$ & Shaanxi & 2007 & Su11-10 & $2,3,4,6,7,8,9,11,14$ & - & - \\
\hline $07-5-6-9$ & Shaanxi & 2007 & Su11-10 & $2,3,4,6,7,8,9,11,14$ & - & - \\
\hline $07-7-1-15$ & Gansu & 2007 & Su11-10 & $2,3,4,6,7,8,9,11,14$ & - & - \\
\hline \multicolumn{7}{|c|}{ Isolates used in validation of sequence characterized amplified region markers } \\
\hline $08-5-11-2$ & Shaanxi & 2008 & CYR22 & $1,2,3,4,5,6,7,8,9,10,11,13$ & - & - \\
\hline $09-7-2-3$ & Gansu & 2009 & CYR22 & $1,2,3,4,5,6,7,8,9,10,11,13$ & - & - \\
\hline $07-6-2-1$ & Sichuan & 2007 & CYR22 & $1,2,3,4,5,6,7,8,9,10,11,13$ & - & - \\
\hline $08-6-2-2$ & Sichuan & 2008 & CYR22 & $1,2,3,4,5,6,7,8,9,10,11,13$ & - & - \\
\hline $09-6-3-1$ & Sichuan & 2009 & CYR22 & $1,2,3,4,5,6,7,8,9,10,11,13$ & - & - \\
\hline $07-5-6-5$ & Shaanxi & 2007 & CYR24 & $1,2,3,4,6,7,8,9,11$ & - & - \\
\hline $08-5-4-9$ & Shaanxi & 2008 & CYR24 & $1,2,3,4,6,7,8,9,11$ & - & - \\
\hline $09-5-7-2$ & Shaanxi & 2009 & CYR24 & $1,2,3,4,6,7,8,9,11$ & - & - \\
\hline $06-5-2-5$ & Shaanxi & 2006 & CYR29 & $1,2,3,4,5,6,7,8,9,11,12,16$ & - & - \\
\hline $08-5-4-3$ & Shaanxi & 2008 & CYR29 & $1,2,3,4,5,6,7,8,9,11,12,16$ & - & - \\
\hline $09-5-4-1$ & Shaanxi & 2009 & CYR29 & $1,2,3,4,5,6,7,8,9,11,12,16$ & - & - \\
\hline $07-7-2-2$ & Gansu & 2007 & CYR29 & $1,2,3,4,5,6,7,8,9,11,12,16$ & - & - \\
\hline $08-7-1-5$ & Gansu & 2008 & CYR29 & $1,2,3,4,5,6,7,8,9,11,12,16$ & - & - \\
\hline $09-7-2-1$ & Gansu & 2009 & CYR29 & $1,2,3,4,5,6,7,8,9,11,12,16$ & - & - \\
\hline $08-6-8-7$ & Sichuan & 2008 & CYR29 & $1,2,3,4,5,6,7,8,9,11,12,16$ & - & - \\
\hline $09-6-8-5$ & Sichuan & 2009 & CYR29 & $1,2,3,4,5,6,7,8,9,11,12,16$ & - & - \\
\hline $08-6-4-10$ & Sichuan & 2008 & CYR30 & $1,2,3,4,5,6,7,8,9,11,12,16,17$ & - & - \\
\hline $07-5-3-13$ & Shaanxi & 2007 & CYR31 & $1,2,3,4,5,6,7,8,9,11,12,14,16,17$ & - & - \\
\hline $08-5-2-9$ & Shaanxi & 2008 & CYR31 & $1,2,3,4,5,6,7,8,9,11,12,14,16,17$ & - & - \\
\hline $08-7-5-14$ & Gansu & 2008 & CYR31 & $1,2,3,4,5,6,7,8,9,11,12,14,16,17$ & - & - \\
\hline $09-7-1-11$ & Gansu & 2009 & CYR31 & $1,2,3,4,5,6,7,8,9,11,12,14,16,17$ & - & - \\
\hline $08-6-4-5$ & Sichuan & 2008 & CYR31 & $1,2,3,4,5,6,7,8,9,11,12,14,16,17$ & - & - \\
\hline $09-6-6-13$ & Sichuan & 2009 & CYR31 & $1,2,3,4,5,6,7,8,9,11,12,14,16,17$ & - & - \\
\hline $06-5-2-18$ & Shaanxi & 2006 & CYR32 & $1,2,3,4,5,6,7,8,9,10,11,12,13,14,16,17$ & + & - \\
\hline $07-5-7-3$ & Shaanxi & 2007 & CYR32 & $1,2,3,4,5,6,7,8,9,10,11,12,13,14,16,17$ & + & - \\
\hline $08-5-1-12$ & Shaanxi & 2008 & CYR32 & $1,2,3,4,5,6,7,8,9,10,11,12,13,14,16,17$ & + & - \\
\hline $09-5-6-10$ & Shaanxi & 2009 & CYR32 & $1,2,3,4,5,6,7,8,9,10,11,12,13,14,16,17$ & + & - \\
\hline $06-7-1-5$ & Gansu & 2006 & CYR32 & $1,2,3,4,5,6,7,8,9,10,11,12,13,14,16,17$ & + & - \\
\hline $07-7-10-2$ & Gansu & 2007 & CYR32 & $1,2,3,4,5,6,7,8,9,10,11,12,13,14,16,17$ & + & - \\
\hline $08-7-12-1$ & Gansu & 2008 & CYR32 & $1,2,3,4,5,6,7,8,9,10,11,12,13,14,16,17$ & + & - \\
\hline $09-7-5-11$ & Gansu & 2009 & CYR32 & $1,2,3,4,5,6,7,8,9,10,11,12,13,14,16,17$ & + & - \\
\hline $06-6-10-11$ & Sichuan & 2006 & CYR32 & $1,2,3,4,5,6,7,8,9,10,11,12,13,14,16,17$ & + & - \\
\hline $07-6-8-2$ & Sichuan & 2007 & CYR32 & $1,2,3,4,5,6,7,8,9,10,11,12,13,14,16,17$ & + & - \\
\hline $08-6-8-5$ & Sichuan & 2008 & CYR32 & $1,2,3,4,5,6,7,8,9,10,11,12,13,14,16,17$ & + & - \\
\hline $09-6-4-3$ & Sichuan & 2009 & CYR32 & $1,2,3,4,5,6,7,8,9,10,11,12,13,14,16,17$ & + & - \\
\hline $06-5-13-10$ & Shaanxi & 2006 & CYR33 & $1,2,3,4,5,6,7,8,9,10,11,12,13,14,16$ & - & + \\
\hline $07-5-13-8$ & Shaanxi & 2007 & CYR33 & $1,2,3,4,5,6,7,8,9,10,11,12,13,14,16$ & - & + \\
\hline $08-5-12-6$ & Shaanxi & 2008 & CYR33 & $1,2,3,4,5,6,7,8,9,10,11,12,13,14,16$ & - & + \\
\hline $09-5-12-4$ & Shaanxi & 2009 & CYR33 & $1,2,3,4,5,6,7,8,9,10,11,12,13,14,16$ & - & + \\
\hline $06-7-3-13$ & Gansu & 2006 & CYR33 & $1,2,3,4,5,6,7,8,9,10,11,12,13,14,16$ & - & + \\
\hline $07-7-2-6$ & Gansu & 2007 & CYR33 & $1,2,3,4,5,6,7,8,9,10,11,12,13,14,16$ & - & + \\
\hline $08-7-1-2$ & Gansu & 2008 & CYR33 & $1,2,3,4,5,6,7,8,9,10,11,12,13,14,16$ & - & + \\
\hline $09-7-1-8$ & Gansu & 2009 & CYR33 & $1,2,3,4,5,6,7,8,9,10,11,12,13,14,16$ & - & + \\
\hline $06-6-1-10$ & Sichuan & 2006 & CYR33 & $1,2,3,4,5,6,7,8,9,10,11,12,13,14,16$ & - & + \\
\hline $07-6-5-2$ & Sichuan & 2007 & CYR33 & $1,2,3,4,5,6,7,8,9,10,11,12,13,14,16$ & - & + \\
\hline $08-6-2-6$ & Sichuan & 2008 & CYR33 & $1,2,3,4,5,6,7,8,9,10,11,12,13,14,16$ & - & + \\
\hline $09-6-16-2$ & Sichuan & 2009 & CYR33 & $1,2,3,4,5,6,7,8,9,10,11,12,13,14,16$ & - & + \\
\hline $07-6-1-7$ & Sichuan & 2007 & Su11-4 & $2,3,4,6,7,8,9,10,11,13,14,16$ & - & - \\
\hline $09-6-1-6$ & Sichuan & 2009 & Su11-4 & $2,3,4,6,7,8,9,10,11,13,14,16$ & - & - \\
\hline $06-5-4-3$ & Shaanxi & 2006 & Su11-7 & $1,2,3,4,5,6,7,8,9,11,12,14,16$ & - & - \\
\hline $07-5-5-2$ & Shaanxi & 2007 & Su11-7 & $1,2,3,4,5,6,7,8,9,11,12,14,16$ & - & - \\
\hline $08-5-4-6$ & Shaanxi & 2008 & Su11-7 & $1,2,3,4,5,6,7,8,9,11,12,14,16$ & - & - \\
\hline $09-5-10-1$ & Shaanxi & 2009 & Su11-7 & $1,2,3,4,5,6,7,8,9,11,12,14,16$ & - & - \\
\hline $07-7-3-6$ & Gansu & 2007 & Su11-7 & $1,2,3,4,5,6,7,8,9,11,12,14,16$ & - & - \\
\hline $08-7-5-2$ & Gansu & 2008 & Su11-7 & $1,2,3,4,5,6,7,8,9,11,12,14,16$ & - & - \\
\hline
\end{tabular}


conditions were inoculated with a mixture of urediniospores of selected isolates with talc. Mock inoculated plants with only talc served as the negative check. The conditions of growing plants before and after inoculation and the inoculation procedure were the same as previously described $(2,13)$. Leaf samples were collected every day from 1 day after inoculation to before sporulation. Genomic DNA was extracted from each leaf sample using the procedure described below for isolating DNA from $P$. striiformis f. sp. tritici urediniospores.

DNA extraction. DNA from each isolate was extracted using the following procedures. Briefly, $20 \mathrm{mg}$ of urediniospores were ground with $200 \mathrm{mg}$ of sand. The powder was suspended in $600 \mu \mathrm{l}$ of lysis buffer $(50 \mathrm{mM}$ Tris, $\mathrm{pH} 8.0,50 \mathrm{mM}$ EDTA, $2 \%$ sodium dodecyl sulfate, $1 \%$ mercaptoethanol, and $0.1 \mathrm{mg} / \mathrm{ml}$ of proteinase $\mathrm{K}$ ) and incubated at $65^{\circ} \mathrm{C}$ for 40 min. The lysate was extracted with an equal volume of phenol and then with an equal volume of chloroform/isoamyl alcohol (24:1). Ammonium acetate was added to a final $1 \mathrm{M}$ concentration and gently mixed, and the DNA was precipitated by adding 2 volumes of dehydrated ethanol and centrifuged for $15 \mathrm{~min}$ at maximum speed in a microcentrifuge $(12,000 \times g)$. The DNA pellet was washed in $70 \%$ ethanol, dried, and resuspended in $50 \mu \mathrm{l}$ of water.

PCR amplifications. Amplification with RAPD primers was performed in a thermal cycler (GeneAmp PCR System 9700). The primers were synthesized decamers from Shanghai Biotech Inc. (Shanghai, China). A total of 94 primers were screened for their ability to produce consistent RAPD pat- terns. These primers were assessed on a subset of $P$. striiformis f. sp. tritici isolates selected based on their genetic diversity, geographic origin, and virulence. The amplifications were performed in a total volume of $25 \mu$ containing $2 \mathrm{mM} \mathrm{MgCl} 2,200$ $\mu \mathrm{M}$ each deoxynucleoside triphosphate, 0.2 $\mu \mathrm{M}$ each primer, $50 \mathrm{ng}$ of genomic DNA, and 1.0 unit of DNA polymerase (Huamei Biotech, Beijing, China). The amplification conditions were the following: a denaturation step for $5 \mathrm{~min}$ at $94^{\circ} \mathrm{C}$ followed by 40 amplification cycles consisting of $30 \mathrm{~s}$ at $94^{\circ} \mathrm{C}, 30 \mathrm{~s}$ at $36^{\circ} \mathrm{C}$, and $90 \mathrm{~s}$ at $72^{\circ} \mathrm{C}$. A final extension step was performed for 7 $\min$ at $72^{\circ} \mathrm{C}$. The PCR products were run on $1.2 \%$ agarose gels, and the DNA was visualized under UV light after staining in an ethidium bromide solution $(0.5 \mu \mathrm{g} / \mathrm{ml})$ for $30 \mathrm{~min}$.

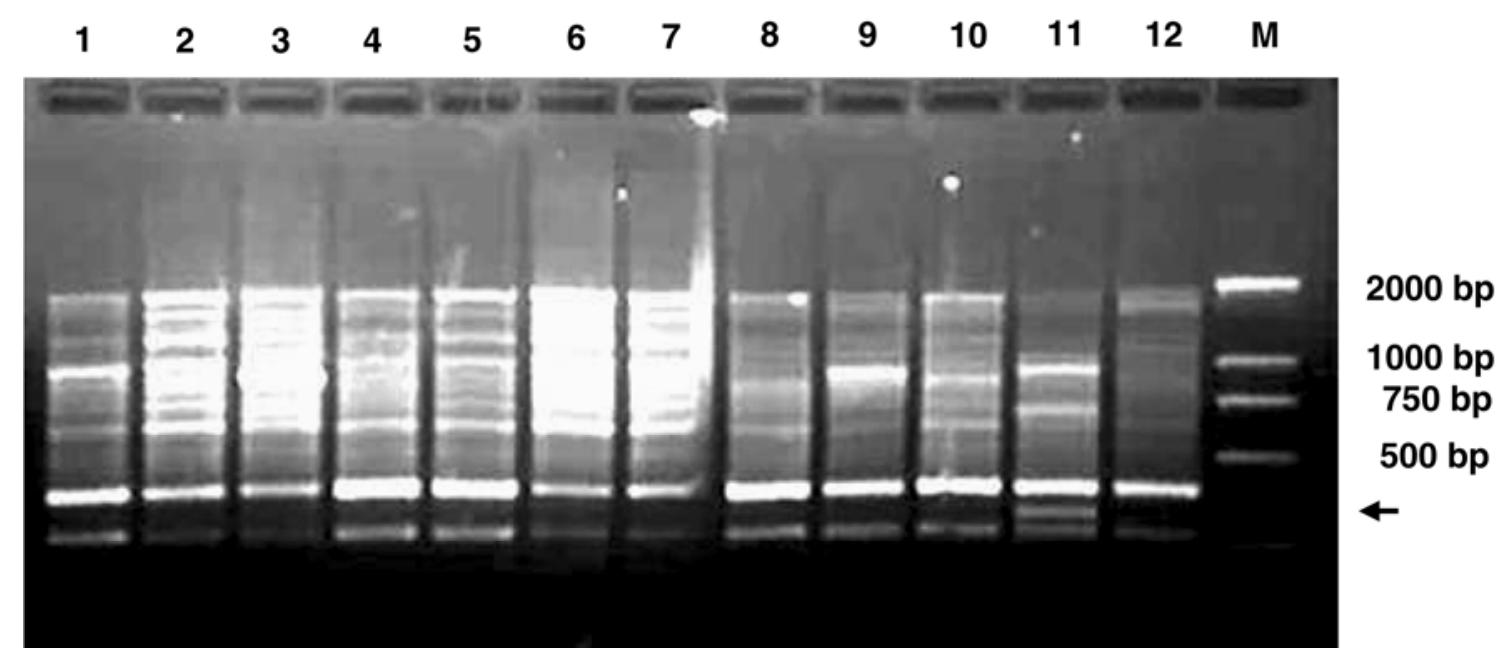

Fig. 1. Agarose gel showing presence and absence of a random amplified polymorphic DNA (RAPD) marker in 12 isolates of Puccinia striiformis f. sp. tritici representing 12 races amplified with primers S1271. The arrow shows the specific RAPD marker, a 320-bp band only present in an isolate of race CYR32. Lane M: DNA ladder DL2000. Lanes 1-12 are isolates for races Su11-1, Su11-3, Su11-4, Su11-5, Su11-6, Su11-7, Su11-10, CYR33, CYR29, CYR31, CYR32, and Hy46-8, respectively.

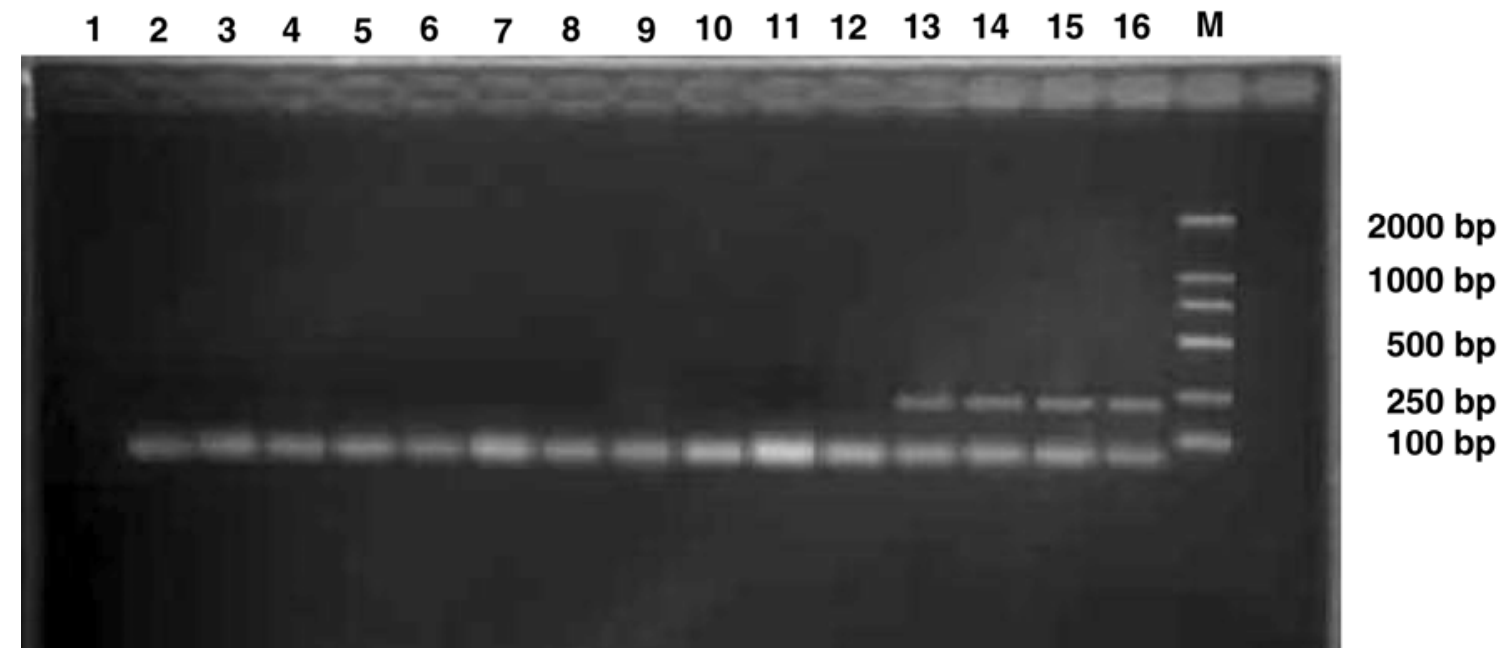

Fig. 2. Agarose gel showing presence of a sequence characterized amplified region (SCAR) marker, CYR32sp1/sp2, in wheat leaves inoculated with race CYR32 and absence of the marker in urediniospore DNA of wheat leaves infected by other races of Puccinia striiformis f. sp. tritici. Lane M: DNA ladder DL2000; lane 1: genomic DNA of wheat genotype Mingxian 169 mock inoculated with talc; lanes 2-5: total genomic DNA of urediniospores of race CYR22, CYR24, CYR31, and CYR33, respectively; lanes 6-12: total genomic DNA of Mingxian 169 infected by CYR22, CYR24, CYR29, CYR31, CYR33, Su11-1, and Su11-3, respectively; lanes 13-16: total genomic DNA of Mingxian 169 inoculated with isolates of CYR32 collected from Taibai, Baoji, and Yangling of Shaanxi Province and Sichuan Province, respectively. 
DNA cloning and sequencing. Amplified DNA fragments were separated on a $1.5 \%$ agarose gel. The race-specific bands were purified from gels using the Agarose Gel Extraction Kit (Roche Inc., Germany) and cloned into the pGEM-T-easy vector (Promega, USA). Ligations, transformations of Escherichia coli, and plasmid amplifications were performed following the standard procedures (18). After cloning, 20 positive colonies from each transformation were selected, and each colony was cultured overnight in a Luria-Bertani (LB) liquid medium containing ampicillin at $100 \mathrm{mg} / \mathrm{liter}$. The size of the cloned RAPD fragments was verified by PCR using the corresponding RAPD primers or universal primers $\mathrm{T} 3$ and $\mathrm{T} 7$, and digested by the restriction enzymes in the multiple clone site of the vector. The recombinant plasmids were extracted using the alkaline lysis method (1), and fragments were sequenced with an ABI 373A automated DNA sequencer (Jierui Biotech, Shanghai, China). Sequences were assembled using ClusterX 2.0 and deposited in the GenBank of the National Center for Biotechnology Information (NCBI) with accession numbers EU404159 and GQ241245.

Design of SCAR primers and PCR detection of $P$. striiformis f. sp. tritici races. Primers for candidate SCAR markers were designed using the Primer 3 software. For each cloned RAPD fragment, two pairs of primers were designed from each sequence. The primers were synthesized by Jierui Biotech. The amplification of the SCAR markers was done with reaction mixtures similar to those for the RAPD assay, except that $2.5 \mathrm{mM} \mathrm{MgCl} \mathrm{m}_{2}$ and 0.6 units of $\mathrm{Taq}$ polymerase were used. The primers for the amplification of SCAR markers were CYR32sp1 (5'-CGCTAT GTGCGGACAGAAGG-3') and CR32sp2 (5'-TGAGCATCAAGCCTATGAGG-3') for race CYR32; and CYR33sp1 (5'TGTCGTCTCGCCAATCTTT-3') and CYR33sp2 (5'-GCGGGTGTCAGTTTC TCC-3') for CYR33. The amplification conditions were the same as those described above, except that the annealing temperature was $57^{\circ} \mathrm{C}$ for primers CR32sp1/CY32sp2 and $56^{\circ} \mathrm{C}$ for primers CYR33sp1/CYR33sp2. All PCR reactions with SCAR primers were repeated at least three times, and included the negative (water or wheat leaf DNA) and positive (1 ng of plasmid DNA with corresponding RAPD fragment) controls. The PCR products were checked on agarose gels as described above.

\section{RESULTS}

RAPD polymorphisms. In this study, the 94 tested RAPD primers produced polymorphic and distinguishable bands. The sizes of bands selected as RAPD markers varied from 0.3 to $2.0 \mathrm{~kb}$ and were consistently amplified in three PCR replicates to confirm the reproducibility of the polymorphism. A total of 2,949 RAPD bands were amplified, of which 911 (approximately $31 \%$ ) were polymorphic among the tested isolates. The number of bands per primer ranged from 1 to 14 . In general, 3 to 4 polymorphic bands were detected with each RAPD primer, and banding profiles were different among the isolates.

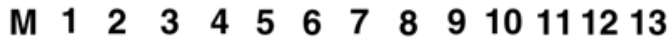

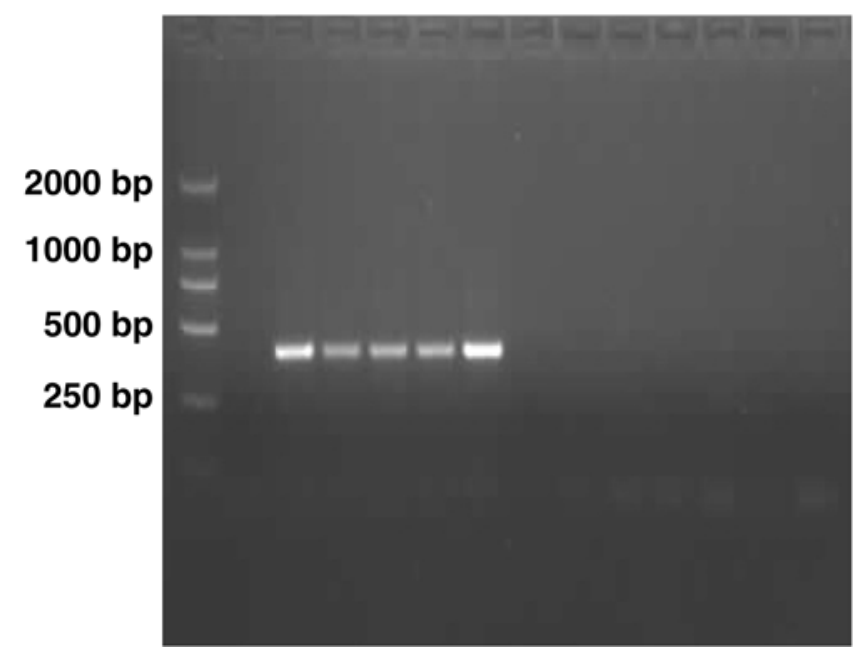

Fig. 4. Agarose gel showing presence of a sequence characterized amplified region (SCAR) marker, CYR33sp1/sp2, in wheat leaves infected with isolates of race CYR33 and absence of the marker in urediniospores of and wheat leaves infected with isolates of other races of Puccinia striiformis $\mathrm{f}$. sp. tritici. Lane M: DNA ladder DL2000; lane 1: genomic DNA of wheat genotype Mingxian169 mock inoculated with talc; lanes 2-4: genomic DNA of Mingxian 169 infected with isolates of CYR33 from Shaanxi, Sichuan, and Gansu provinces, respectively; lane 5 and 6: genomic DNA from single spores of CYR33; and lanes 7-13: urediniospore genomic DNA of CYR 22, CYR24, CYR29, CYR31, CYR32, Su11-3, and Su11-10, respectively.

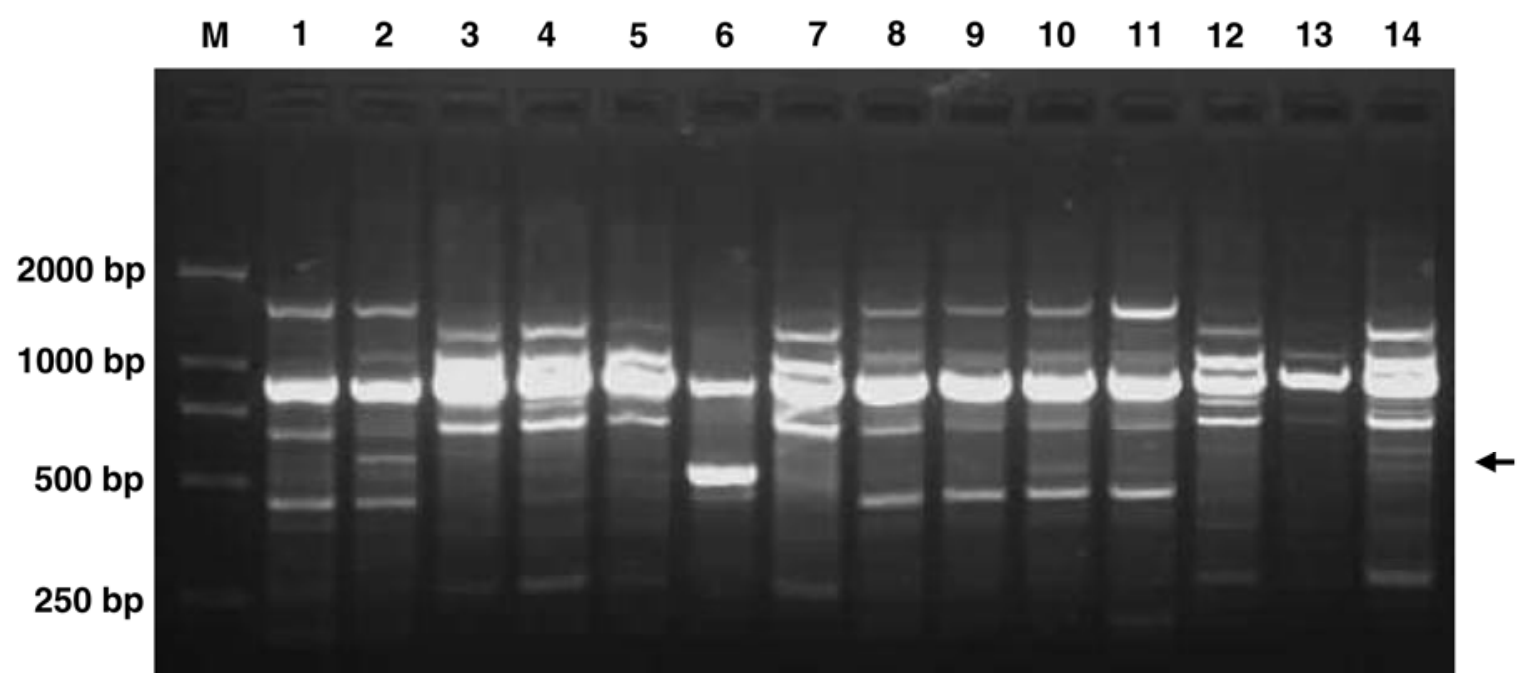

Fig. 3. Agarose gel showing presence of a random amplified polymorphic DNA (RAPD) marker amplified with primer S1340 in an isolate of race CYR33 and absence in the other 13 isolates representing 13 races of Puccinia striiformis $\mathrm{f}$. sp. tritici. The arrow shows the specific RAPD marker, a 550-bp band in a CYR33 isolates. Lane M: DNA ladder DL2000. Lanes 1-14: CYR32, CYR31, CYR29, CYR24, CYR22, CYR33, Su11-1, Su11-3, Su11-4, Su11-5, Su11-6, Su11-7, Su11-10, and Hy46-8, respectively. 
Development of SCAR markers. Two of the RAPD markers specifically associated with races CYR32 and CYR33 were selected and converted to the SCAR markers. A 320-bp fragment associated with CYR32 was generated by primer S1271 (5'-CTTCTCGGTC-3') (Fig. 1). This specific band was presented in 10 single-spore isolates and repeatable in three PCR replicates. The band was cloned and seven clones were sequenced. The seven clones produced an identical sequence, which was deposited in the GenBank as accession number EU404159. Based on this sequence, two primers were designed: CYR32SP1: 5'-CGCTATGTGCGGACA GAAGG-3' and CYR32SP2: 5'-TGAGGA TCGAGGCTATGAGG-3'. PCR amplifications with this primer pair and DNA from the above-mentioned isolates as templates were performed. The expected 240-bp fragment was present in all isolates of race CYR32 only (Fig. 2), indicating that the SCAR marker CYR32sp1/sp2 was specific for race CRY32. The CYR32-specific DNA fragment was cloned and sequenced, and the sequence confirmed that it was the expected part of the original RAPD S1271 marker.

A RAPD band of 550 bp produced by primer S1304 (5'-AGGAGCGACA-3') distinguished isolates of race CYR33 from others (Fig. 3). To develop a SCAR marker for CYR33, the specific band was cloned and sequenced. The sequence was deposited in GenBank as GQ241245. Based on the sequence, two primers were designed: CYR33sp1 (5'-TGTCGTCTCGCCAAT CTTT-3') and CYR33sp2 (5'-GCGGGT GTCAGTTTCTCC-3'). When the five single-spore isolates of CYR33 and other isolates of Su11 races were used as PCR templates, the primer pair produced the expected 397-bp band in all isolates of CYR33, but not in other isolates (Fig. 4). Thus, the SCAR marker CYR33sp1/sp2 was specific for race CYR33.

PCR detection of CYR32 and CYR33 in wheat leaves using the SCAR markers. To test the efficiency and specificity of the SCAR markers for detecting races CYR32 and CYR33, PCR reactions with the SCAR marker primers were performed using leaves of susceptible wheat genotype Mingxian 169 inoculated with isolates of the two races collected from different provinces. The leaves were sampled every day after inoculation until sporulation, and the genomic DNA samples were used as templates for PCR. The CYR32sp1/sp2 and CYR33sp1/sp2 markers were detected in DNA samples from leaves from 5 days after inoculation with isolates of CYR32

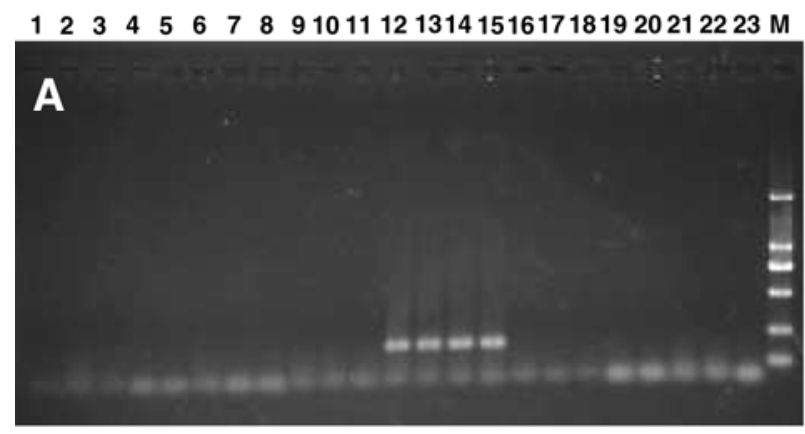

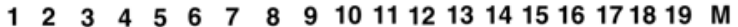

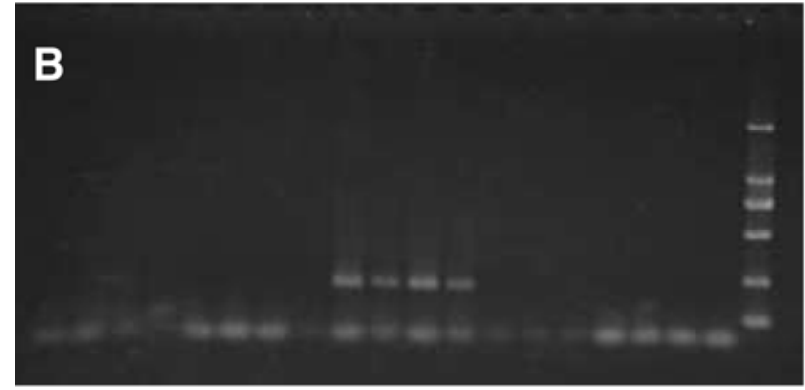

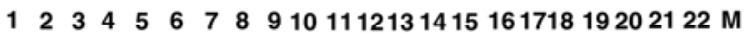

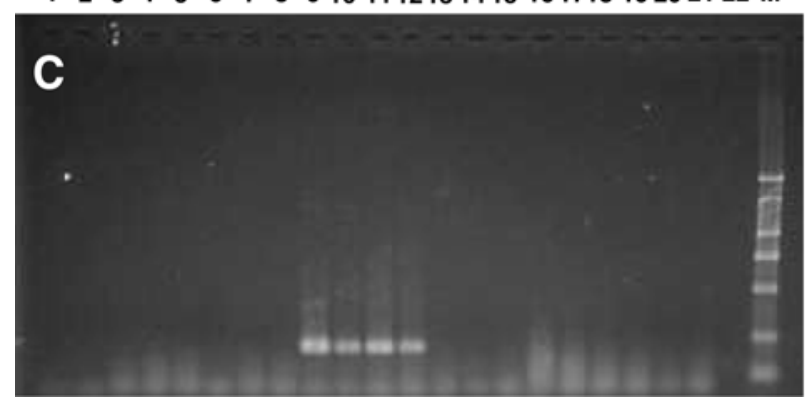

$12234456678891011121314151617181920212223 \mathrm{M}$

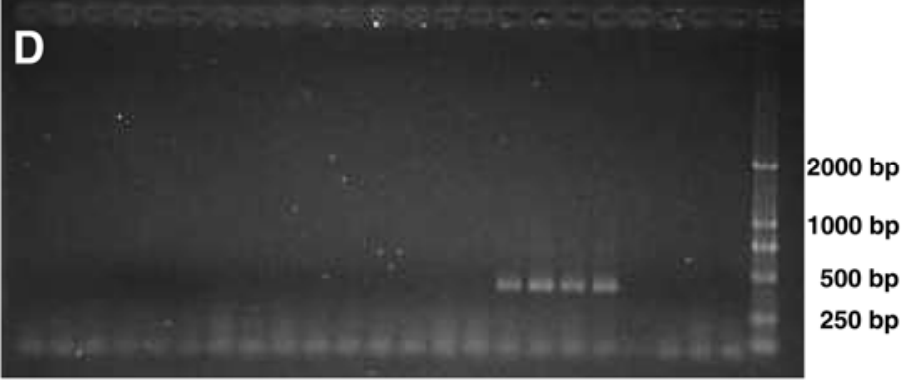

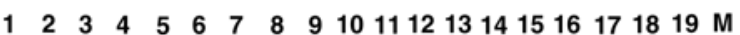

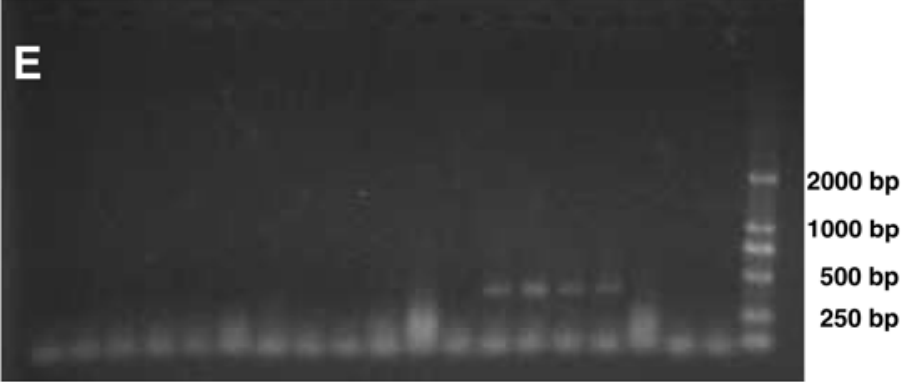

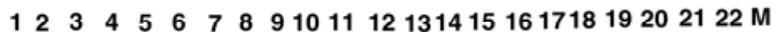

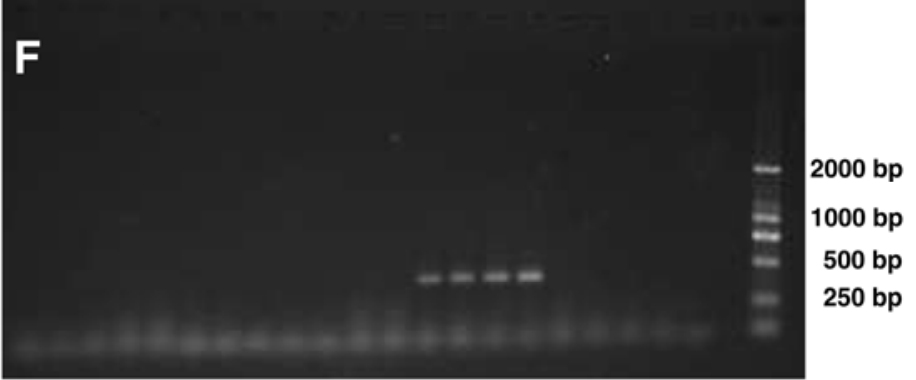

Fig. 5. Agarose gels showing the presence of a sequence-characterized amplified region (SCAR) marker in urediniospores of CYR32 (A, B, C) and CYR33 (D, E, F) collected from Shaanxi, Gansu, and Sichuan in different years. A and D, Lanes 1-23: 08-5-11-2 (CYR22), 09-5-2-2 (CYR22), 07-5-6-5 (CYR24), 08-5-4-9 (CYR24), 09-5-7-2 (CYR24), 06-5-2-5 (CYR29), 08-5-4-3 (CYR29), 09-5-4-1 (CYR29), 07-5-3-13 (CYR31), 08-5-2-9 (CYR31), 09-5-4-15 (CYR31), 06-5-2-18 (CYR32), 07-5-7-3 (CYR32), 08-5-1-12 (CYR32), 09-5-6-10 (CYR32), 06-5-13-10 (CYR33), 07-5-13-8 (CYR33), 08-5-12-6 (CYR33), 09-5-12-4 (CYR33), 06-5-4-3 (Su11-7), 07-5-5-2 (Su11-7), 08-5-4-6 (Su11-7), and 09-5-10-1 (Su11-7); lane M: DNA ladder DL2000. B and E, Lanes 1-19: 08-7-2-6 (CYR22), 09-7-2-3 (CYR22), 07-7-2-2 (CYR29), 08-7-1-5 (CYR29), 09-7-2-1 (CYR29), 07-7-1-6 (CYR31), 08-7-5-14 (CYR31), 097-1-11 (CYR31), 06-7-1-5 (CYR32), 07-7-10-2 (CYR32), 08-7-12-1 (CYR32), 09-7-5-11 (CYR32), 06-7-3-13 (CYR33), 07-7-2-6 (CYR33), 08-7-1-2 (CYR33), 09-7-1-8 (CYR33), 07-7-3-6 (Su11-7), 08-7-5-2 (Su11-7), and 09-7-12-2 (Su11-7); lane M: DNA ladder DL2000. C and F, Lanes 1-22: 07-6-2-1 (CYR22), 08-6-2-2 (CYR22), 09-6-3-1 (CYR22), 08-6-8-7 (CYR29), 09-6-8-5 (CYR29), 08-6-4-10 (CYR30), 08-6-4-5 (CYR31), 09-6-6-13 (CYR31), 066-10-11 (CYR32), 07-6-8-2 (CYR32), 08-6-8-5 (CYR32), 09-6-4-3 (CYR32), 06-6-1-10 (CYR33), 07-6-5-2 (CYR33), 08-6-2-6 (CYR33), 09-6-16-2 (CYR33), 07-6-1-7 (Su11-4), 09-6-1-6 (Su11-4), 07-6-3-1 (Su11-7), 09-6-5-4 (Su11-7), 08-6-8-3 (Su11-11), and water; lane M: DNA ladder DL2000. 
and CYR33, respectively. Plants inoculated with water or other isolates did not have any of the markers (Figs. 2 and 4).

Comparison of PCR detection with SCAR markers and virulence test with differentials. In order to evaluate the usefulness and validate the SCAR markers for detecting races CYR32 and CYR33, 63 isolates of $P$. striiformis $\mathrm{f}$. sp. tritici races, which were collected from Shaanxi, Gansu, and Sichuan provinces from 2006 to 2009 , were identified using both differential test and detection with the SCAR markers. As expected, DNA samples from all isolates of CYR32 produced the CYR32sp1/sp2 band, and all isolates of CYR33 produced the CYR33sp1/sp2 band. Isolates of all other races (CYR22, CYR24, CYR29, CYR31, Su11-4, Su11-7, and Su11-11) did not produce any of the marker bands (Table 1 and Fig. 5). The results of the SCAR marker detections completely matched those of the race tests. These results showed that the SCAR markers were reliable for identification of races CYR32 and CYR33 and faster than virulence testing.

\section{DISCUSSION}

In China, the first publication on $P$. striiformis f. sp. tritici race identification was in 1944 (9). Later, Fang and Chen (10) identified 9 races using 7 wheat and 1 barley genotypes. Several differential sets were used in the following decade. Since 1957, the Chinese National Wheat Rust Collaborative Group (CNWRCG) has been coordinating the standard wheat genotypes of the differential set and keeping the race identification identical (14,25-28). The current differential set consists of 17 wheat genotypes $(21,23)$. Based on the virulence patterns on the differential set, specific isolates of the pathogen have been named either race or "pathotype" based on frequencies of virulence patterns. Usually, a pathotype referred to a particular virulence pattern with a very low frequency in the population. If the frequency of a particular pathotype increases to a certain level in the population, it will be redesignated as a race. For example, the pathotype Su1114 was first identified in 1997 with a frequency of $1.2 \%$. In recent years, the frequency of Su11-14 has increased, and therefore the CNWRCG named Su11-14 as CYR33 in 2008 (4). In all, the CNWRCG identified 71 races and pathotypes by 2008. In the current population, CYR32 and CYR33 have more broad virulence spectra than other races or pathotypes. CYR32 is virulent to all 17 differentials except Zhong 4, which carries a resistance gene from Thinopyrum intermedium (21). CYR33 is virulent to all 17 differentials except Zhong 4 and Hybrid 46. Rapid detection of these two races should provide important information for disease management.
The objective of this study was to develop a race-specific, highly reliable PCRbased detection assay for detecting CYR32 and CYR33. The RAPD bands associated with these races were sequenced from isolates from diverse geographic regions and found to be suitable target loci for designing race-specific primers. The CYR33sp1/sp2 marker amplified with primers CYR33sp1/ sp2 was dominant and only present in isolates of CYR33. The primers CYR32sp1/ sp2 amplified two fragments, the CYR32sp1/sp2 band was unique to CYR32 and the other smaller band was present in all $P$. striiformis f. sp. tritici isolates. These SCAR markers were shown to be reliable for detecting CYR32 and CYR33.

Stripe rust is able to develop quickly into an epidemic in most wheat growing areas of China. There are few commercial cultivars resistant to the currently prevalent races, CYR32 and CYR33. Monitoring the dynamics of these races is an important task for control of stripe rust. However, the current race identification takes at least 2 weeks, and it is impossible to identify races using the traditional differential test before the pathogen sporulates. The delayed detection of races may result in missing the critical time for fungicide application or unnecessary application as some cultivars are resistant to one of the predominant races but not the other. The two new SCAR markers can shorten race identification to 1 day and are able to detect CYR32 and CYR33 from infected leaves before sporulation. Together with the previously identified markers $(2,13)$, races CYR29, CYR31, CYR32, and CYR33 can be more efficiently monitored using the PCR approach. Markers for other races should be developed in order to have a clear picture of race composition in the pathogen virulence monitoring in the early season of stripe rust.

\section{ACKNOWLEDGMENTS}

The study was supported by the 111 project from Education Ministry of China (No. B07049), and Key Projects in the National Science \& Technology Pillar Program during the Eleventh Five-year Plan Period (2006BAD08A05).

\section{LITERATURE CITED}

1. Ausubel, F. M., Brent, R., Kingston, R. E., Moore, D. D., Seidman, J. G., Smith, J. A., and Struhl, K. 2002. Short Protocols in Molecular Biology, 5th ed. John Wiley \& Sons, New York.

2. Cao, L. H., Kang, Z. S., Zheng, W. M., Huang, L. L., and Li, Z. Q. 2005. The development of SCAR detection marker of Puccinia striiformis f. sp. tritici race CYR31 in China. Mycosystema 24:98-103.

3. Chen, C. Q., Zheng, W. M., Buchenauer, H., Huang, L. L., Lu, N. H., and Kang, Z. S. 2009. Isolation of microsatellite loci from expressed sequence tag library of Puccinia striiformis $\mathrm{f}$. sp. tritici. Mol. Ecol. Res. 9:236-238.

4. Chen, W. Q., Wu, L. R., Liu, T. G., Xu, S. C., Jin, S. L., Peng, Y. L., and Wang, B. T. 2009. Race dynamics, diversity, and virulence evolution in Puccinia striiformis f. sp. tritici, the causal agent of wheat stripe rust in China from 2003 to 2007. Plant Dis. 93:1093-1101.
5. Chen, X. M. 2005. Epidemiology and control of stripe rust [Puccinia striiformis $\mathrm{f}$. sp. tritici] on wheat, Can. J. Plant Pathol. 27:314-337.

6. Chen, X. M., Line, R. F., and Leung, H. 1993. Relationship between virulence variation and DNA polymorphism in Puccinia striiformis. Phytopathology 83:1489-1497.

7. Chen, X. M., Line, R. F., and Leung, H. 1995. Virulence and polymorphic DNA relationships of Puccinia striiformis f. sp. hordei to other rusts. Phytopathology 85:1335-1342.

8. Enjalbert, J., Duan, X., Giraud, T., Vautrin, D., de Vallavieille-Pope, C., and Solignac, M. 2002. Isolation of twelve microsatellite loci, using an enrichment protocol, in the phytopathogenic fungus Puccinia striiformis f. sp. tritici. Mol. Ecol. Notes 2:563-565.

9. Fang, C. T. 1944. Physiological specialization of Puccinia glumarum Erikss. and Henn. In China. Phytopathology 34:1020-1024.

10. Fang, C. T., and Chen, N. Y. 1955. Varietal resistance of wheat to stripe rust at the seedling stage and the variability of causal organism. Acta Phytopathol. Sinica 1:155-168. (In Chinese with English abstract.)

11. Hovmøller, M. S., Yahyaoui, A. H., Milus, E. A., and Justesen, A. F. 2008. Rapid global spread of two aggressive strains of wheat rust fungus. Mol. Ecol. 17:3818-3826.

12. Justesen, A. F., Ridout, C. J., and Hovmøller, M. S. 2002. The recent history of Puccinia striiformis f. sp. tritici in Denmark as revealed by disease incidence and AFLP markers. Plant Pathol. 51:13-23.

13. Kang, Z. S., Cao, L. H., Zheng, W. M., Huang, L. L., and Li, Z. Q. 2005. Selection and foundation of a race specific molecule marker for wheat stripe rust race CY29 in China. J. Northwest Sci-tech Univ. Agric. For. 33:54-56. (In Chinese with English abstract.)

14. Li, Z. Q., Wang, M. N., Jia, M. G., Lu, H. P., Shang, H. S., Kang, Z. S., Yang, S. F., Sun, S. M., and Shi, Y. C. 1997. Study of the epidemiology and control strategy of wheat stripe rust in South Gansu. Acta Univ. Agric. BorealiOccidentalis 25:1-5.

15. Li, Z. Q., and Zeng, S. M. 2002. Wheat Rusts in China. China Agricultural Press, Beijing. pp. 1-11. (In Chinese.)

16. Mboup, M., Leconte, M., Gautier, A., Wan, A., Chen, W., de Vallavieille-Pope, C., and Enjalbert, J. 2009. Evidence of genetic recombination in wheat yellow rust populations of a Chinese oversummering area. Fungal Genet. Biol. 46:299-301

17. Newton, A. C., Caten, C. E., and Johnson, R. 1985. Variation for isozyme and doublestranded RNA among isolates of Puccinia striiformis and two other cereal rusts. Plant Pathol. 34:235-247.

18. Sambrook, J., and Russell, D. W. 2001. Molecular Cloning: A Laboratory Manual, 3rd ed Cold Spring Harbor Laboratory, Cold Spring Harbor, NY.

19. Shan, W., Chen, S., Kang, Z. S., Wu, L., and Li, Z. 1998. Genetic diversity in Puccinia striiformis Westend. f. sp. tritici revealed by pathogen genome-specific repetitive sequence. Can. J. Bot. 76:587-595.

20. Szabo, L. J., and Kolmer, J. 2007. Development of simple sequence repeat markers for the plant pathogenic rust fungus Puccinia triticina. Mol. Ecol. Notes 7:708-710.

21. Wan, A. M., Chen, X. M., and He, Z. H. 2007 Wheat stripe rust in China. Aust. J. Agric. Res. 58:605-619.

22. Wan, A. M., Niu, Y. C., Wu, L. R., Yuan, W. H., Li, G. B., Jia, Q. Z., Jin, S. L., Yang, J. X., Li, Y. F., and Bi, Y. Q. 1999. Physiologic specialization of wheat stripe rust in China during 1991-1996. Acta Phytopathol. Sinica 29:15-21. (In Chinese with English abstract.)

23. Wan, A. M., Zhao, Z. H., Chen, X. M., He, Z H., Jin, S. L., Jia, Q. Z., Yao, G., Yang, J., 
Wang, B. T., Li, G. B., Bi, Y. Q., and Yuan, Z. Y. 2004. Wheat stripe rust epidemic and virulence of Puccinia striiformis f. sp. tritici in China in 2002. Plant Dis. 88:896-904.

24. Wang, B. T., Li, G. B., Li, Q., Wang, F., Shi, Y. Q., Liu, Q. R., and Kang, Z. S. 2009. Parasitic fitness of the major epidemic strains of Puccinia striiformis $\mathrm{f}$. sp. tritici in China. Acta Phytopathol. Sinica 39:82-87. (In Chinese with English abstract.)

25. Wang, F. L., Wu, L. R., Xu, S. C., Jin, S. L., Jia, Q. Z., Yuan, W. H., and Yang, J. X. 1996. The discovery and studies on new races
CYR30 and CYR31 of wheat stripe rust in China. Plant Prot. 23:40-44. (In Chinese with English abstract.)

26. Wang, K. N., Hong, X. W., Si, Q. M., Wang, J. X., and Shen, J. P. 1963. Studies of the physiological specialization of stripe rust of wheat in China. Plant Prot. 2:23-35. (In Chinese with English abstract.)

27. Wang, K. N., Hong, X. W., Wang, J. X., Meng, Q. Y., Xie, S. X., Wu, L. R., and Cheng, M. R. 1985. Studies of the physiological specialization of stripe rust of wheat in China during 1962-1965 and 1972-1974. Bull. Inst. Plant
Prot. 1:31-36

28. Wang, K. N., Wu, L. R., Meng, Q. Y., Xie, S X., Lu, D. Y., Yuan, W. H., Yu, X. Y., Song, W. Z., Liu, S. J., Wang, J. Q., Yang, J. X., Huang, G. M., Li, Y. F., and Yang, S. C. 1986. On the physiological specialization of stripe rust of wheat in China during the year 1975-1984. Acta Phytopathol. Sinica 16:79-85. (In Chinese with English abstract.)

29. Wellings, C. R., and McIntosh, R. A. 1990. Puccinia striiformis f. sp. tritici in Australia: Pathogenic changes during the first 10 years. Plant Pathol. 39:316-325. 